\title{
Tsafon
}

Revue d'études juives du Nord

$76 \mid 2018$

Expressions yiddish de la nature

\section{Prier dans les bois et les champs. Israël Baal Shem Tov et la nature}

Jean Baumgarten

\section{(2) OpenEdition}

1 Journals

Édition électronique

URL : https://journals.openedition.org/tsafon/1190

DOI : $10.4000 /$ tsafon. 1190

ISSN : 2609-6420

Éditeur

Association Jean-Marie Delmaire

Édition imprimée

Date de publication : 1 décembre 2018

Pagination : $27-50$

ISSN : 1149-6630

\section{Référence électronique}

Jean Baumgarten, «Prier dans les bois et les champs. Israël Baal Shem Tov et la nature », Tsafon [En ligne], 76 | 2018, mis en ligne le 30 juin 2019, consulté le 23 juin 2021. URL : http:// journals.openedition.org/tsafon/1190; DOI : https://doi.org/10.4000/tsafon.1190

Tsafon. Revues d'études juives du Nord 


\section{Prier dans les bois et les champs Israël Baal Shem Tov et la nature}

\section{Jean Baumgarten *}

À la fin du Guide des égarés ${ }^{1}$, Maïmonide consacre un chapitre au culte divin (avoda), à la connaissance et à l'amour de Dieu (ahava). Il distingue différentes catégories hiérarchisées de croyants. Au bas de l'échelle spirituelle se trouvent les incultes, qui sont dans l'erreur, et les « ignorants qui s'occupent des pratiques religieuses », mais sans les comprendre. Il mentionne ensuite les casuistes, dont ceux qui traitent de sciences mathématiques et de logique, sans toutefois s'engager dans la vérité des principes de la religion. Un dernier degré est composé de ceux qui se sont « rapprochés de la certitude ». Maïmonide explique qu'ils « se consacrent entièrement à Dieu, s'éloignent de tout ce qui est en dehors de Lui » et «sont arrivés dans l'intérieur de la demeure auprès du souverain ». Ce degré est, entre autres, celui des prophètes. Certains parmi eux ont une perception très forte du divin; ils « savent s'isoler de tout ce qui est en dehors de lui » au point d'avoir annihilé les facultés matérielles du corps. Reclus dans «la solitude et l'isolement», ils s'approchent de Dieu, interrogent, parlent et reçoivent sa parole. Le chapitre continue sur la récitation du Shema. Maïmonide, là encore, différencie ceux qui prient mécaniquement et ceux qui s'appliquent à réfléchir intensément sur ce qu'ils prononcent et à comprendre le sens des bénédictions. Un ultime distinguo est établi : il existe un temps pour

\footnotetext{
${ }^{*}$ CNRS-EHESS, Paris.

${ }^{1}$ Maïmonide, Guide des égarés, édité par Salomon Munk, Lagrasse, Verdier, 1979, II, 51. (Dorénavant GE, II, 51).
} 
les choses mondaines, le "régime domestique et les besoins corporels », et un temps pour les choses religieuses, qui suppose d'être tout entier concentré à « s'approcher de Dieu ». Le sujet n'est pas seulement aimant (ohev), mais passionné (hoshek) de Dieu. Ce niveau constitue le degré suprême du lien entre la divinité et sa créature. Chez eux, la violence de l'amour est si forte qu'ils éprouvent une véritable jouissance de Dieu. Maïmonide conclut ainsi le Guide des égarés par la description d'une échelle spirituelle de la croyance, depuis l'approche exotérique, propre à la masse, jusqu'à la connaissance ésotérique, sorte d'au-delà de la pensée rationnelle et intellectuelle. Ce degré supérieur de totale dévotion à Dieu et d'expérience intense du divin ne peut être réservé qu'à une toute petite élite de sages et de savants.

Il est possible de faire une lecture mystique de ce chapitre qui décrit le degré ultime de la connaissance divine propre aux prophètes et aux visionnaires qui ont accès aux secrets de la Torah (Sitrei Torah ${ }^{2}$ ). Notons, de même, que dans ce texte de Maïmonide, la présence de la nature est, sinon absente, tout du moins négligée, effacée, refoulée. Ainsi, plus on monte dans la gradation spirituelle, plus les données somatiques sont mises en veilleuse et jugées négativement. Elles sont essentiellement un facteur d'empêchement de l'association avec le divin. Enfin, dans cette échelle spirituelle, le vulgaire, par opposition à l'élite intellectuelle des initiés ${ }^{3}$, est incapable de s'approcher des connaissances ésotériques. Ces réflexions de Maïmonide ont profondément imprégné un vaste courant de la philosophie juive et de la pensée rabbinique. On en discerne de multiples échos chez de nombreux auteurs qui développeront une vision similaire fondée sur la hiérarchisation de la connaissance du divin $^{4}$.

Certaines doctrines religieuses, notamment la tradition mystique juive, se sont radicalement démarquées de la scolastique rationaliste médiévale ${ }^{5}$ Ces courants vont de la mystique juive ancienne, dont la

\footnotetext{
${ }^{2}$ GE, I, 37, II, 2. Sur le rapport complexe entre 1'ésotérisme de Maïmonide et la mystique juive, voir Moshe Idel, Maïmonide et la mystique juive, Paris, Cerf, 1991, p. 11-60.

${ }^{3}$ Maïmonide fait une distinction entre les «fils d'Elohim », ceux qui sont, par leur intelligence, proches de Dieu et les « fils d'hommes », la masse du vulgaire.

${ }^{4}$ Sur les relations de la philosophie juive et de la kabbale, voir Georges Vajda, Recherches sur la philosophie et la kabbale dans la pensée juive du Moyen Âge, Paris, Mouton, 1962.

${ }^{5}$ Cette assertion demanderait, bien sûr, à être nuancée du fait que la kabbale est traversée par des courants contradictoires. Les écrits d'Abraham Aboulafia, par exemple, se placent résolument dans la filiation intellectuelle de Maïmonide, qu'il
} 
littérature des heykhalot (Palais divins), de la kabbale de Safed, jusqu'au hassidisme ${ }^{6}$. Gershom Scholem y voit le point d'aboutissement du long cheminement de la mystique juive ${ }^{7}$, centré sur la praxis religieuse, la piété, la magie, la kabbale pratique, la démonologie et la possession. Ces aspects avaient été longtemps refoulés comme faisant partie d'un judaïsme marginal, sauvage, pétri de superstitions, axé sur les miracles, les croyances dites populaires, le surnaturel, les états altérés de la conscience, comme dans la prière extatique. Une dichotomie s'est ainsi établie entre les courants intellectuels, les pratiques instaurées par la tradition des rabbins et des autorités instituées, versus un judaïsme $\grave{a}$ l'état de nature ou à l'état sauvage. Cette division apparaît d'autant plus problématique que magie et religion sont constamment intriquées.

Un des points fondamentaux de divergence par rapport aux courants de la philosophie rationaliste juive concerne la conception de la nature qui constitue une des données centriques de la pensée du Baal Shem $\operatorname{Tov}^{8}$. La nature ${ }^{9}$, en hébreu teva ${ }^{10}$, est le point de convergence de

considère comme son maître. Moïse Cordovero, par ailleurs, soutient, comme Maïmonide et comme dans la théologie négative, qu'aucun attribut positif ne peut servir à définir Dieu. Si Cordovero défend, comme les philosophes maïmonidiens, l'idée d'une absolue transcendance divine, par contre, il explique que la relation entre Dieu et sa créature est médiatisée par les sefirot. Cette conception est rejetée par les philosophes médiévaux pour qui Dieu est l'Un et ne peut être séparé en dix attributs. D'autres traités kabbalistiques, à l'inverse, nient les présupposés de la philosophie maïmonidienne, notamment en ce qui concerne la représentation de Dieu qui possède une structure anthropomorphique, soit une correspondance entre le corps humain et le corps divin. Voir Gregg Stern, Philosophy and Rabbinic Culture, Jewish Interpretation and Controversy in Medieval Languedoc, London, Routledge, 2009, p. 143-145.

${ }^{6}$ Peter Schäfer, Le Dieu caché et révélé, introduction à la mystique ancienne, Paris, Cerf, 1993.

${ }^{7}$ Gershom Scholem, Les Grands courants de la mystique juive, Paris, Payot, 1973, p. 343-368.

${ }^{8}$ Gershom Scholem explique à ce sujet : «Où se situe la frontière qui sépare les facultés imaginatives du sens de la réalité (même du paranormal) chez quelqu'un ? C'est loin d'être clair. Pour parler franchement, à mon humble avis, l'histoire de la religion ne pourrait que grandement bénéficier de l'exploration du supranormal ou des visions propres à la parapsychologie ; l'usage conventionnel du terme 'imagination' 'suggestion' et 'autosuggestion' ne révèle pas la vérité de la question dans la mesure où cela est censé tout expliquer sans rien expliquer complètement... ». Voir G. Scholem, « Demuto ha-historit shel R. Yisrael Baal Shem Tov », Molad, 18, 1960, p. 335-356.

${ }^{9}$ En hébreu, homer ha-tiv'i (Lit. : «substance naturelle ») pour désigner tout ce qui concerne la nature, le monde matériel et physique. À quoi s'ajoutent les données intellectuelles, spirituelles, de l'âme (neshama) et de l'esprit (ruah ). Sur la nature dans le judaïsme, voir Hava Tirosh-Samuelson, "Nature in the Sources of Judaism », Daedalus, 130/4, 2001, p. 99-124 ; Judaism and Ecology: Created World and Revealed Word, ed. by H. Tirosh-Samuelson, Cambridge, Harvard University Press, 2002. 
deux axes fondamentaux du judaïsme et, tout particulièrement, de la pensée du Besht. Elle renvoie, en premier lieu, à la relation verticale de l'Alliance (brit) entre Dieu et le peuple d'Israël qui implique des obligations légales, des responsabilités éthiques, ainsi que des rituels, des prières et des pratiques mystiques afin de connecter le monde terrestre et le royaume d'en haut. La nature est, d'autre part, associée au lien horizontal entre les êtres humains et la nature que Dieu a créée. Relation qui est, d'ailleurs, suggérée, selon le principe de la guematria, dans le terme de teva (nature) qui possède la même valeur numérique que elokim (Dieu). La corrélation entre le divin et le monde naturel implique un engagement des humains afin de ne pas dégrader l'œuvre de la création voulue par Dieu, comme l'affirme le Midrash :

Au moment où le Saint, béni soit-il, créa le premier homme, il le prit et lui montra tous les arbres du Jardin d'Eden, puis lui dit: Vois mes œuvres! Vois comme elles sont plaisantes et louables ! Or tout ce que j'ai créé, je l'ai créé pour toi. Garde-toi donc d'endommager ou de détruire mon monde, car si tu l'endommages, il n'y aura personne pour le réparer après toi. ${ }^{11}$

D'autre part, de même que la nature est le vêtement, la demeure, le tabernacle et le Temple de Dieu, de même le corps est un des lieux de rencontre, d'affleurement, avec le divin. Dieu a créé la nature et quiconque veut sentir, goûter et se rapprocher de la présence divine peut le faire, entre autres, en contemplant la beauté de la terre. Si la nature (teva) est souvent opposée à la culture (tarbut), ces deux dimensions ne sont, en fait, que les deux facettes d'une même réalité, qui doivent être associées, sans heurt, au sein d'une demeure commune, la terre. De même que le microcosme et le macrocosme sont en harmonie constante, le milieu naturel et les productions culturelles doivent s'agencer, sans disjonction. Face à une architecture dualiste du monde qui instaure une séparation entre le matériel et le spirituel, dans la pensée mystique, le corps, la matière et l'âme, l'esprit s'interpénètrent, sans qu'il faille postuler une séparation rigide entre les deux composantes de l'être humain, qui ne sont que les deux versants d'une substance unique ${ }^{12}$.

\footnotetext{
${ }^{10}$ À l'origine, teva désigne une pièce de monnaie, la moitié d'un sela, ainsi qu'un élément, une substance. Ce terme n'apparaît pas dans la Bible, où le texte ne fait qu'exalter la beauté du monde. Par la suite, ce terme, en hébreu, désignera la nature.

${ }^{11}$ Kohelet Rabba 7, 13. Selon le principe talmudique: Al tash hit, (Ne détruis pas ! N'abîme pas!).

${ }^{12}$ Cet aspect différencie la pensée philosophique médiévale et la mystique juive. Maïmonide oppose les données somatiques à la faculté intellectuelle. Maïmonide
} 
Nous sommes en présence, pour reprendre le terme de Philippe Descola, d'une anthropologie moniste dans laquelle les lignes de démarcation entre la nature, l'humain et le divin sont mouvantes, ductiles ${ }^{13}$.

Dans le monde de l'étude traditionnelle, notamment celui des yeshivot, les maîtres condamnaient l'adoration de la nature, considérée comme une forme d'idolâtrie (avoda zara ${ }^{14}$ ). Ils se concentraient sur l'étude des textes, la prière, l'interprétation de la Loi, comme seule et unique voie pour connaître Dieu, percer ses mystères et comprendre la création divine. Dieu ne réside que dans la Torah et dans elle seule; en dehors des textes saints, l'être humain ne peut pas saisir sa nature transcendante. L'idée qu'il puisse se fonder une relation entre les créatures et Dieu par le biais de la nature relevait d'une forme de paganisme. On trouve un écho de ce déni de l'importance du monde naturel dans la sentence des Pirkei avot $(3,7)$ : « Rabbi Yaakov dit : Celui qui va en chemin, étudie et interrompt son étude pour dire : 'Comme cet arbre est beau, comme ce sillon est beau', l'Écriture le considère comme mettant sa vie en péril ».

Le Besht, à l'inverse, sans nier la centralité de l'étude et l'importance de la synagogue et de la Beyt ha-Midrash [maison d'étude et de prière], déplace le centre de gravité de la pratique juive en le transportant, entre autres, mais pas uniquement, au sein de la nature, comme un des lieux privilégiés de la présence et de la manifestation du divin. Les plantes, les arbres, les fleurs, les animaux ${ }^{15}$, la nature en général, possèdent une étincelle de sainteté divine du fait qu'ils ont été

explique : « Je dis donc que les parties de l'âme sont au nombre de cinq : la nutritive, la sensitive, l'imaginative, l'attractive et l'intellectuelle ». Il ajoute : « Or, sache que cette âme, qui est une et dont nous venons de décrire les facultés ou les parties, est en quelque sorte la matière et que l'intelligence en est la forme; et tant que cette forme n'est pas devenue son partage, l'existence de l'aptitude à recevoir la forme est comme nulle et sans but [...]. Une âme dépourvue de raison n'est rien de bon ». Dans la pensée mystique, les réalités matérielles sont, entre autres, le locus du divin.

${ }^{13}$ Philippe Descola, Par-delà nature et culture, Paris, Gallimard, Coll. Folio Essais, 2005 ( $1^{\mathrm{e}}$ édition) 2016 ( $2^{\mathrm{e}}$ édition), p. 17.

${ }^{14}$ Sur ce terme se référer au traité du Talmud Avoda Zara. Le troisième chapitre de la Mishna aborde la question des images, des idoles, des divinités païennes, du polythéisme. La déesse Asherah est, par exemple, représentée sous la forme d'un arbre qui devient un objet de culte. On retrouve cette association entre Asherah et les arbres dans différents versets bibliques. Voir I Rois $14: 15$ et 23 ; II Rois $16: 3-4$ et $17: 10$.

${ }^{15}$ Il conviendrait - autre sujet - de parler du rapport entre les animaux et les humains dans la tradition juive. Voir Natan Slifkin, Man and Beast: Our Relationships with Animals in Jewish Law and Thought, Jerusalem, Zoo Torah, 2006. 
créés par Dieu ${ }^{16}$. Communiquer avec les éléments naturels équivaut, de ce fait, à établir une médiation avec le monde divin. Ces liens de connivence ont, de plus, une fonction théurgique. Ils permettent de drainer les influx divins vers le monde terrestre et d'influer sur les sphères supérieures. Être au contact avec les éléments naturels prédispose au dialogue intime avec le Créateur, notamment par la concentration, la prière et la méditation solitaire. Bien plus, chaque sujet - et c'est une dimension essentielle de la pensée hassidique - est une parcelle, un atome au sein d'un réseau complexe de relations qui se déploient dans la totalité de l'univers. Chaque élément de ce vaste système interconnecté renvoie à une structure réticulaire dans laquelle les passerelles, les liens, les correspondances jouent un rôle fondamental. Ce ne sont pas les points de liaison dans ce maillage complexe qui s'avèrent primordiaux, mais bien plus les convergences, les analogies et les connexions. Le lien entre les humains et la nature n'est qu'un des aspects essentiels de ce réseau d'affinités qui structure l'ensemble de la réalité terrestre et du monde divin. On pourrait aussi décliner d'autres correspondances, par exemple, entre le corps et l'univers, les organes somatiques et le corps astral, les membres et les planètes, les caractères, les émotions et les signes zodiacaux. Et ainsi de suite, presque à l'infini.

Ajoutons que Israël Baal Shem Tov, même s'il est conscient des niveaux de culture religieuse, n'établit pas de distinction étanche entre les savants et la masse des fidèles. On pourrait, pour paraphraser Spinoza, dire que, pour lui, « le peuple et les doctes partagent la même fortune $»^{17}$. L'ignorant, tout comme le savant, peut s'associer à Dieu, notamment au sein de la nature, certes selon des modalités différentes, mais sans qu'une coupure étanche existe entre les deux. Une de ses idées fondamentales consiste dans la circulation entre le somatique et le spirituel, la combinaison entre le naturel et le divin, sans qu'il faille arbitrairement les diviser.

Le Baal Shem Tov, conjointement, s'éloigne de l'idée d'une absolue transcendance du divin qui se situerait dans un au-delà radicalement inaccessible aux humains. Pour Maïmonide, par exemple, il

\footnotetext{
${ }^{16}$ Dans le roman de Chaim Potok The Chosen (New York, 1966, p. 108), on lit ces lignes représentatives de la légende du Besht: « He would walk under the trees, look at the flowers, sit by a brook, listen to the songs of the birds and to the noise of the wind in the leaves. As often as his teachers brought him back, so often did he run away to these woods, and after a while they gave up and left him alone »

${ }^{17}$ Spinoza, Traité Théologico-Politique, édité par Charles Appuhn, Paris, GarnierFlammarion, 1965, chap. VII, p. 156.
} 
existe un abîme infranchissable entre humains et Dieu. Pour les hassidim, la prière Écoute, Israël : l'Éternel est notre Dieu, l'Éternel est un! (Deut $6,4)$ témoigne, tout au contraire, de l'unité du divin qui imprègne la moindre parcelle de la création. Un des disciples du Besht, Pinhas de Koretz explique encore : «Le terme un ( $\mathrm{e} h \mathrm{ad}$ ) suppose qu'il n'y a rien d'autre dans la terre entière que le Saint, béni soit-Il qui emplit toute la terre de Sa gloire ${ }^{18} .$. Il n'y a rien dans tout le monde, excepté le Dieu un $\gg{ }^{19}$.

Dans la pensée du Besht, on trouve de multiples témoignages sur son goût de la nature. Il aimait à s'isoler dans les vastes forêts de sa région natale, les Carpates. Certes, il s'agit d'une pratique ancienne dont on trouve d'innombrables traces dans les textes saints. Dès les premiers chapitres de la Bible, le texte enseigne que s'instaure entre Dieu, l'être humain et la nature une alliance fondée sur la responsabilité et l'obligation de protéger et de faire fructifier la terre : «L'Éternel-Dieu prit donc l'homme et l'établit dans le jardin d'Eden pour le cultiver (leovda) et le soigner (ule-shomra) ${ }^{20}$. L'idée centrale est de faire bon usage de la nature, sans la dégrader, ni la ruiner, au risque de détruire le pacte qui unit le Créateur et sa créature. Les textes canoniques développent l'idée de l'harmonie entre la créature humaine, la nature et les animaux, qui complémente l'Alliance entre Dieu et l'humanité. Maïmonide peut ainsi expliquer :

Quelle est la voie pour atteindre l'amour et la crainte de Dieu ? Quand une personne contemple (hitbonen) ses grandes, merveilleuses œuvres et créations,

\footnotetext{
${ }^{18}$ Isaïe 6,3 .

${ }^{19}$ Idée qu'on retrouve dans les Tikkunei ha-Zohar, $57:$ «Il n'y a aucun espace vide de Lui ». Il n'y a pas d'ange dans lequel ne se trouve le nom YHWH, car ce nom se trouve en tout lieu, comme l'âme se trouve en chaque membre (du corps). C'est pourquoi l'homme doit faire régner YHWH en toute sefira et en tout Trône, en tout ange et en tout membre de l'homme, car aucun lieu n'est vide de Lui, ni dans les (mondes) supérieurs, ni dans les (mondes) inférieurs. YHWH n'est invoqué dans l'unification des quatre lettres qu'en vertu de la Cause des causes qui les unifie et parce que c'est Lui (la Cause des causes, soit l'Eyn sof, l'infini) qui unifie les quatre lettres, c'est en Lui que sont invoquées (les quatre lettres) YHWH par l'unification une : « Le Seigneur est Un et son Nom est Un » (Dt 6, $4 ; \mathrm{Za} \mathrm{14,9).} \mathrm{C'est} \mathrm{pour} \mathrm{cela} \mathrm{que} \mathrm{la} \mathrm{foi} \mathrm{d'Israël} \mathrm{s'exprime} \mathrm{de}$ manière adéquate par ces quatre lettres. Et tous les noms (autres que ce nom) sont des noms de remplacement de ce nom. Il n'y a pas de nom jusqu'à l'infini et jusqu'à l'inachevé qui soit plus grand et plus puissant que ce nom, en haut jusqu'à l'infini et en bas jusqu'à l'inachevé. Et toutes les armées et les cohortes (d'anges) tremblent et sont ébranlées devant Lui ».

${ }^{20}$ Gen 2,15.
} 
qu'elle voit son immense, infinie sagesse, immédiatement, elle aimera, louera, vantera et désirera ardemment connaître le Grand Nom. ${ }^{21}$

Une multitude de pratiques, de prières, de rituels, témoignent de la force du lien entre Dieu, les humains et la nature ; le cycle des fêtes est, ainsi, associé au rythme de la nature ${ }^{22}$. Nombre de bénédictions remercient Dieu pour ses bienfaits, que ce soit la beauté de la terre, la saveur des fruits, la variété des plantes et des semences : "Béni sois-tu, Oh Dieu, maître de l'univers, qui a créé Son monde dans lequel rien ne manque, qui y a créé de bonnes créatures, de bons arbres odoriférants, pour donner du plaisir à l'humanité $»^{23}$. Rappelons également que durant la période talmudique, la société juive n'était pas encore fortement urbanisée. De nombreux sages, savants et rabbis étaient des fermiers qui vivaient et étudiaient au contact de la nature, comme en témoignent les traités du Talmud de Jérusalem sur l'agriculture ${ }^{24}$.

Bien plus, c'est au sein de la nature qu'ils sentaient, goûtaient et savouraient la présence divine. L'idée centrale de la perception de la grandeur divine au sein de la nature sera déclinée par un grand nombre de penseurs juifs, notamment dans la littérature éthique (Sifrut ha-musar) et surtout dans la mystique juive. Le sentiment de la nature y est particulièrement développé, entraînant une recherche du contact intime avec les éléments comme moyen de s'associer au Créateur, de sentir sa majesté et, par une forme de fusion dans l'immensité cosmique, de s'extraire des pesanteurs terrestres et d'élever son âme vers les sphères supérieures. Ainsi, chez les mystiques de Safed, la présence divine imprègne tous les éléments de la terre qu'il faut respecter et chercher à connaître pour sentir et percevoir le divin. Isaac Luria avait ainsi l'habitude de s'isoler, d'aller étudier à l'ombre des oliviers, d'aller

\footnotetext{
${ }^{21}$ Maïmonide, Mishneh Torah, Hilkhot Yesodei ha-Torah, 2, 2. On observe une complémentarité entre les diverses perceptions du divin chez Maïmonide. Il décrit le Dieu transcendant, perçu par la contemplation intellectuelle, et le Dieu dont chaque croyant doit chercher, sentir et goûter la présence, notamment au sein de la beauté du monde. De même, le philosophe déprécie les réalités somatiques et le médecin recherche l'équilibre entre le corps et l'âme. Sur l'amour de Dieu chez Maïmonide, voir Georges Vajda, L'Amour de Dieu dans la théologie du Moyen Âge, Paris, Vrin, 1957, p. 118-140.

${ }^{22}$ Rappelons quelques bénédictions liées à la nature : quand on voit un arc-en-ciel (zokher ha-brit), quand on admire les merveilles de la nature (oseh ma'aseh bereshit), l'océan (she-asah et-ha-yam ha-gadol), des arbres (she kakhah lo be-olamo)... Citons également, entre autres, la fête des arbres (Tu bi-shvat, rosh ha-shanah shel etsim).

${ }^{23}$ Talmud de Babylone, Berakhot $43 \mathrm{~b}$ et $54 \mathrm{~b}$.

${ }^{24}$ Dans le Talmud de Jérusalem, voir les Seder Zera'im dont la plupart des traités portent sur l'agriculture.
} 
méditer auprès des tombes des Amoraïm et des Tannaïm de l'époque talmudique, afin d'entrer en contact avec eux ${ }^{25}$. Déjà, vers 1560 , alors qu'il vivait en Égypte, il s'imposa un complet isolement durant six ans sur une petite île le long du Nil. Six jours par semaine, sauf le shabbat, il s'extrayait du monde pour s'absorber dans la prière et les pratiques contemplatives $^{26}$. De même, chez son élève, Moïse Cordovero, dans le Palmier de Deborah, on peut lire :

Il faut que l'homme honore toutes les créatures parce qu'en elles il connaîtra la hauteur du Créateur qui forma l'homme avec sagesse; de même toutes les créatures recèlent en elles la sagesse du Formateur. Qu'il voie de lui-même qu'elles sont, en effet, honorables à l'extrême, car le Formateur de tout, le sage exalté au-dessus de tout, a pris soin de les créer. Et s'il les méprise - Dieu pardonne - il porte atteinte à l'honneur de leur Formateur. ${ }^{27}$

Le Besht, s'inscrit visiblement dans cette tradition mystique qui recherche le contact avec la nature. L'essentiel de sa vie se passa dans un environnement rural de villages, hameaux et shtetlekh au contact des paysans. Durant son enfance, des récits racontent qu'il étudiait au heder [l'école primaire juive] et que, durant des jours, il s'évadait. Les habitants de son village le recherchaient; ils finissaient par le retrouver dans la forêt où il aimait rester, solitaire, au sein de la nature. On attribuait ces évasions au fait qu'il était orphelin. Il devint l'assistant (bahelfer) du maître d'école (melamed) chargé, entre autres, de conduire les enfants à la synagogue et de leur apprendre les prières. Quand il marchait en leur compagnie, il chantait avec une voix plaisante qui montait jusqu'aux cieux et les anges se réjouissaient. Un récit ${ }^{28}$ explique que, de peur que les chants si purs du Besht ne viennent accélérer la disparition du Mal et la venue du Messie, le Satan se métamorphosa en sorcier, puis en loup-garou. Il attaqua et effraya les enfants qui se dispersèrent. Le conte poursuit : «Le Besht se souvint les mots de son

\footnotetext{
${ }^{25}$ Voir le Zohar II, 198b, Tikkunei ha-Zohar, introduction. Le Sefer gerushim (Livre des bannissements, Venise 1600, Jérusalem, Ha-Ivri, 1962) de Moïse Cordovero raconte ces expériences d'errance, d'exil volontaire, pour imiter l'exil de la présence divine et s'associer à Dieu. Voir aussi Le Palmier de Debora, éd. et trad. Charles Mopsik, Lagrasse, Verdier, 1985, p. 111 : «Que l'homme s'exile de lieu en lieu au nom du ciel, en cela il façonnera un char (merkava) pour la Shekhina exilée ».

${ }^{26}$ Lawrence Fine, Physician of the Soul, Isaac Luria and His Kabbalistic Fellowship, Stanford, Standford University Press, 2003, p. 35-37.

${ }^{27}$ Moïse Cordovero, Palmier de Debora, op. cit., chap 2, p. 80.

${ }^{28}$ Voir In Praise of the Baal Shem Tov, Shivhei ha-Besht, éd. par Dan Ben-Amos et Jerome R. Mintz, New York, Schoken, 1984, p. 12.
} 
père, que Dieu le bénisse, de ne jamais avoir peur puisque Dieu était avec lui. Il puisa de la force en Dieu, son Dieu ». Durant la première partie de son expérience spirituelle, il vit reclus, que ce soit dans une "pièce de solitude » (beyt ha-hitbodedut) ou dans des lieux isolés propices à la méditation, la concentration mentale, et les techniques mystiques d'ascension de l'âme, de prière extatique et d'attraction des influx divins. Cet isolement permet d'atteindre la « réalité essentielle » (mehuyav hametsiut) ou la "pureté essentielle », soit une forme d'anéantissement qui provoque la perception du divin ${ }^{29}$. La nature n'est que le « vêtement », la «parure » de la présence divine. Tous les aspects de la réalité dévoilent l'unicité de la création divine ou, comme le dit le Besht: «La nature est l'essence de la déité ${ }^{30}$. D'autre part, en référence à la cosmologie d'Isaac Luria, le Besht pense que des étincelles de sainteté sont disséminées dans tous les éléments de la nature. S'absorber en elle permet ainsi d'extraire ces germes de divinité emprisonnés au sens de la matière, de les libérer et de s'associer à Dieu.

Parallèlement à la synagogue (shul), à l'oratoire (kloyz), au lieu d'isolement (heder meyuhad), pour lui, la nature est un espace sacré où il peut entrer en contact direct avec le divin. Attitude que ses adversaires ${ }^{31}$ ne pouvaient que condamner, d'autant que la communication avec Dieu ne se fait plus par la médiation d'un rabbin au sein de la communauté, par l'étude des textes saints, mais directement, dans la solitude et le silence des forêts et des montagnes ${ }^{32}$. Ce choix fut considéré par nombre d'autorités rabbiniques et communautaires, soit comme un signe de folie, d'égarement, de séparation de la vie juive, soit comme une forme de culte païen et panthéiste dans lequel la nature était sacralisée, voire

\footnotetext{
${ }^{29}$ Voir le Zohar 42b; Menahem Nahum de Chernobyl, Me'or Eynaim, Hayei Sarah, Ma'amar Ve-Avraham Zaken, Slawuta, 1798.

${ }^{30}$ Voir Daniel Matt (ed.), The Essential Kabbalah, Harper San Franciso 1996, p. 24-30. Daniel Matt présente une série de textes sur la connexion entre l'infiniment petit du monde matériel, notamment la nature, et l'immensité infinie du divin. Moshe de Leon, Sefer ha-Rimmon; Moshe Cordovero, Elimah Rabbasi, Shi'ur Koma, et Or Yakar al haZohar ; Pinhas de Koretz, Likutey Amarim 14d (cité dans le Sefer Baal Shem Tov, Va'eshanan, sec. 13) ; Menahem Nahuum de Chernobyl, Me'or Einayim, Noah, s.v. vi-hinei isa'ita shekhinah ba-tahtonim; Shneur Zalman of Liadi, Tanya: Sha'ar ha-Yihud veha-Emunah, chap. 1.

${ }^{31}$ Voir Tsava'at ha-Ribash, New York, Kehot, 1998, 12 : «Quand vous vous rendez compte que le Maître de l'univers est présent dans chaque mot et chaque geste, qu'il soit grand ou petit, toutes les confusions qui éclipsent la lumière de l'esprit sont dissipées $\gg$.

${ }^{32}$ Sur les critiques du hassidisme, voir Mordekhay Wilensky, Hassidim u-Mitnaggedim, Jérusalem, Mossad Bialik, 1970.
} 
divinisée ${ }^{33}$. La différence majeure réside dans l'approche textuelle des opposants au hassidisme (mitnaggedim) pour qui le texte canonique se suffit à lui-même (Torah lishma). Il contient la part de vérité que Dieu a voulu transmettre et c'est à celui qui étudie de la rechercher dans le texte lui-même. Chez les hassidim, il existe un au-delà du texte qui a une vertu transformative, liée à l'expérience mystique. On lit le texte, certes pour soi, mais aussi car il a un pouvoir théurgique et c'est un outil performatif pour s'extraire du monde terrestre et s'associer au divin. C'est sans doute l'une des raisons, parmi d'autres, qui explique la condamnation virulente des mitnaggedim de la pensée des premiers hassidim, concernant notamment la prière extatique, les pratiques rituelles jugées extravagantes, la popularisation de la kabbale, les techniques mystiques, notamment le retrait du monde et l'isolement au sein de la nature ${ }^{34}$.

De nombreux maîtres mirent leurs pas dans ceux du Besht, en prolongeant ses exercices mystiques et ses pratiques théurgiques, notamment par la fusion, voire l'anéantissement, dans l'immensité de la nature. On pense à l'arrière-petit-fils du Besht, Nahman de Bratslav, chez qui nous observons des expériences mystiques similaires, oscillant entre la solitude au milieu de la nature et les expériences extatiques de libération intérieure. Certes, le fondement de sa pensée repose sur des prémices différentes de ceux du Besht ${ }^{35}$. Nous retrouvons, toutefois, nombre d'ingrédients qui caractérisent la doctrine hassidique en général. D'abord, l'esseulement et la méditation mystiques (hitbodedut), soit dans une pièce isolée (heder meyuhad), soit au sein de la nature pour prier, s'associer, voire s'entretenir avec le divin. Ce n'est que par ce retrait absolu du monde, par la suspension du brouhaha, du désordre quotidien, de tout éparpillement parasite, que l'être humain peut sentir la proximité de Dieu $^{36}$ :

\footnotetext{
${ }^{33}$ Se référer à Michael Levine, « Pantheism », The Stanford Encyclopedia of Philosophy (édition de 2012), Edward N. Zalta (ed.), URL https://plato.stanford.edu/archives/sum2012/entries/pantheism/ et William Mander, « Pantheism », The Stanford Encyclopedia of Philosophy (édition de 2016), Edward N. Zalta (ed.), URL

https://plato.stanford.edu/archives/win2016/entries/pantheism/.

${ }^{34}$ Allan Adler, The Faith of the Mithnagdim, Rabbinic Responses to Hasidic Rapture, Baltimore, John Hopkins University Press, 1997, p. 153-164.

${ }^{35}$ Arthur Green, Tormented Master, Woodstock, Jewish Lights, 1992.

${ }^{36}$ Le conte de Nahman de Bratslav intitulé Le Maître de la prière raconte l'histoire d'un juif qui se consacre entièrement à la louange divine : «Le maître de prière s'était établi en un lieu hors de toute habitation où coulait une rivière et poussaient des arbres fruitiers dont il se nourrissait ». Voir Nahman de Bratslav, Contes de Nahman, éd. par Laurent Cohen, Waterloo, Matanel, 2013, p. 161.
} 
Quand l'été approche, c'est bon de méditer dans les champs. C'est un moment où vous pouvez prier Dieu avec désir et envie. La méditation et la prière sont une conversation (siha) (avec Dieu). Un buisson dans un champ est une siha $a$. Quand tous les buissons dans les champs commencent à revenir à la vie et pousser, alors tous aspirent à être associés dans la prière et la méditation. ${ }^{37}$

Nahman de Bratslav pratiquait une forme extrême de la solitude mystique en marchant dans la forêt, allant se recueillir sur la tombe du Besht, son arrière-grand-père, pour prier et, comme il le dit, "parler à Dieu comme s'il était un ami ». On raconte même que, l'hiver, il se roulait dans la neige afin d'éteindre le feu de pulsions parasites (mahshavot zarot) qui l'habitaient ${ }^{38}$. Ses textes comprennent de multiples références à l'importance de s'engloutir, s'abîmer et se perdre dans l'infinité de la nature: "Toutes les plantes et herbes entrent dans votre prière et vous assistent $»{ }^{39}$. La joie d'être dans la nature correspond à la joie de s'unir à Dieu :

Tu dois avoir le privilège d'entendre les chants et louanges des herbes ! Comme chaque brin d'herbe chante une louange à Dieu, sans motivations égoïstes, sans pensées parasites, sans considération de récompense. Comme cela est beau et bon d'entendre leurs chants! Comme il est bon d'être parmi eux et de servir Dieu avec crainte ! Heureux sont ceux qui peuvent prier en forêt : les herbes et les arbres, toutes les plantes élèvent un chant sublime vers leur Créateur. Lorsqu'on se met à prier parmi les plantes, leurs louanges se mêlent à la nôtre et l'élèvent vers le Ciel. Maître de l'univers, offre-moi la possibilité d'être seul. Puisse cela devenir mon habitude d'être dehors chaque jour parmi les arbres, l'herbe, toutes les plantes qui poussent, et ainsi, être seul pour entrer en prière. ${ }^{40}$

La nature, comme le corps humain (ha-olam ha-katan) réverbère les sphères supérieures, soit la terre, les planètes et de plérôme (ha-olam ha-gadol). D'autant que chaque créature - choses, animaux, plantes et humains - possède une graine ou une étincelle de sainteté (nitsotsot hakedusha). Se fondre dans la nature permet ainsi de s'unir au divin, présent dans la moindre parcelle de ce qui a été créé par Dieu lors du processus d'engendrement du monde (ma'aseh bereshit). Cette communion avec les éléments est censée donner un avant-goût du Paradis.

\footnotetext{
${ }^{37}$ Nahman de Bratslav, Hayye Moharan, Jérusalem, Torat ha-netsah, 1996, n 72.

${ }^{38}$ Nah̄man de Bratslav, Shivhei ha-Ran, Jérusalem, Torat ha-netsah, 1991, nº19.

${ }^{39}$ Nah̆man de Bratslav, Likkutei Moharan Jérusalem, 1999, II, p. 11-12.

${ }^{40}$ Nah̆man de Bratslav, Sihot ha-ran, Jérusalem, Torat ha-netsah, 1991, n 163 et 227.
} 
Un autre passage rend bien compte de l'importance d'être au milieu des éléments naturels :

Lorsqu'on devient digne de percevoir le chant des plantes, d'entendre comme chaque brin d'herbe lance son chant vers Dieu, spontanément, que ce chant paraît alors si doux et harmonieux! Voilà pourquoi il est si agréable de servir Dieu en pleine nature, de se promener par les champs, au milieu des produits du sol et de répandre ses propres paroles. Celles de plantes pénètrent alors dans les vôtres et en augmentent la puissance. À chaque respiration, vous aspirez l'air du Gan Eden et lorsque vous rentrez chez vous, le monde se trouve régénéré à vos yeux. $^{41}$

Certains mystiques s'avèrent donc plus aptes à communiquer avec les éléments de la nature. Ils se situent, de ce fait, aux lisières de la vie sociale puisqu'ils quittent souvent le brouhaha de la vie communautaire pour s'isoler au sein des immensités naturelles aux fins d'entrer en conversation avec le divin. Ils sont souvent perçus comme des marginaux, des solitaires pour qui la nature est un refuge propice à leurs expériences mystiques. On comprend ainsi pourquoi les tsaddikim suscitèrent une vive opposition de la part des autorités rabbiniques qui stigmatisaient leur retrait de la société et leurs comportements jugés extravagants, interprétés comme des signes de déviance mentale.

\section{Du monde naturel à la nature humaine}

Il existe, dans la pensée hassidique ${ }^{42}$, une autre acception fondamentale de la notion de nature. Elle désigne, dans ce cas, les dispositions psychophysiques de la nature humaine qui peuvent, dans certains états limites, projeter le sujet dans une dimension supraconsciente, dans des régions inconnues du psychisme, au-delà de ce qu'il a l'habitude de rationnellement appréhender ${ }^{43}$. Ces états se caractérisent

\footnotetext{
${ }^{41}$ Cité par Laurent Cohen, Le Maître des frontières incertaines, Paris, Seuil, 1994, p. 47-48. Voir aussi ce passage : « Le Rebbe disait que c'est une bonne chose pour celui qui veut servir Dieu d'avoir des chevaux. Il peut, de la sorte, aller dans les forêts avec de jeunes compagnons (d'étude) pour discuter sur la crainte de dieu et méditer. Les champs et les forêts sont d'excellents lieux pour la concentration (hitbodedut) »; voir Nahman de Bratslav, Hayyei Moharan, op. cit., 1996, n²30.

${ }^{42}$ Sur les dimensions psychiques de l'expérience mystique des hassidim, le rôle de l'imagination religieuse, se reporter à Zvi Mark, Mysticism and Madness, The Religious Thought of Rabbi Nachman of Bratslav, Londres, Continuum, 2009.

${ }^{43}$ Sur les états limites ou modifiés de la conscience, voir Georges Lapassade, États modifiés de la conscience, Paris, PUF, 1987 ; idem, La Transe, Paris, PUF, 1990.
} 
par des symptômes corporels plus ou moins paroxystiques qui détonent par rapport aux comportements prônés dans le judaïsme rabbinique, marqués par la recherche d'un certain silence du corps, qui doit, notamment lors de la prière, être mis en sourdine, en signe de soumission devant le Créateur. Tout mouvement incontrôlé, désordonné ne peut être qu'un signe d'irrespect et d'absence de concentration. Quant au dérèglement des sens et au relâchement de l'attention, d'autant plus dans un lieu sacré comme une synagogue, ils sont interprétés par les autorités rabbiniques comme une preuve que la personne est dominée par l'esprit de folie (ruah shtut) et d'idolâtrie (avoda zara $)^{44}$. Les hassidim, à l'inverse, ont été de fins explorateurs des profondeurs de la psyché humaine. En référence à la tradition mystique ancienne, à la kabbale d'Isaac Luria, aussi bien que des techniques de la kabbale pratique, de la magie, ils se sont particulièrement attachés à la décrire et vivre les états modifiés de la conscience, à élargir leur horizon mental en vue de s'unir au divin.

L'intercesseur entre les forces terrestres et célestes joue, dans cette perspective, un rôle essentiel. C'est à lui qu'incombe la tâche fondamentale de relier le haut et le bas, de faire descendre les influx divins vers la terre, de faire remonter les prières vers les hauteurs célestes et de veiller à ce que les canaux de circulation des énergies ne soient pas obstrués. On a souvent comparé sa fonction à celle d'un chaman ${ }^{45}$ qui peut faire monter son âme vers le plérôme pour entrer en contact avec les âmes des morts et qui sert de médiateur entre les différents niveaux de la création divine. Le $b a^{\text {'al }}$ Shem ${ }^{46}$, le maître du Saint Nom, a en charge la gestion des flux d'énergie qui régule les rituels, accompagne la pratique des commandements et ainsi participe à l'équilibre entre les membres de la communauté et entre les forces du haut et du bas, entre les vivants et les morts. Il a en cela un rôle pacificateur de contrôle, de régulation et d'incitation. En même temps qu'il désamorce les conflits, les différends, les tensions qui risquent de perturber les liens horizontaux entre les membres de la communauté et la communication verticale entre les hommes, les anges et Dieu. C'est un conciliateur grâce à qui les éléments, que certains courants de la pensée juive tendaient à dissocier,

\footnotetext{
${ }^{44}$ Moshe Idel, « La folie dans la pensée juive », Les Cahiers du judaïsme, 13, 2003, p. 4-19; Zvi Mark, op. cit., 2009.

${ }^{45}$ Roberte Hamayon (dir.), Chamanismes, Paris, PUF, 2003.

${ }^{46}$ Immanuel Etkes, " Magic and Miracle Workers in the Day of the Baal Shem Tov », The Besht, Magician, Mystic and Leader, Waltham, Brandeis University Press, 2005, p. 7-45.
} 
s'harmonisent, se complètent, sans qu'il y ait de cassure entre les aspirations humaines et la volonté supérieure de Dieu. La nature tend, de ce fait, à être sacralisée, de même que, dans une certaine mesure, Dieu est humanisé ou anthropomorphisé.

Le Besht est un parfait exemple de ce type de figure mystique chez qui les manifestations extérieures de la piété prennent souvent des formes paroxystiques, qui étaient entrevues par les rabbins comme le signe d'une nature perturbée, voire d'un dérèglement, propres à ceux qu'on nomme, dans les traités mystiques, les fous de Dieu ${ }^{47}$. La plupart des éléments doctrinaux qui singularisent sa pensée et ses pratiques tournent autour de l'association avec le divin par le corps et l'expression d'un embrasement mystique qui conduit aux débridements du comportement que ses adversaires mettaient sur le compte d'une nature corrompue, dominée par le penchant à mal faire (yetser ha-ra). Son comportement dérangé aurait été l'indice d'un lien avec un paganisme idolâtre, que le monothéisme rabbinique aurait tendu à sublimer, discipliner et évacuer ${ }^{48}$.

Les textes attribués au Besht et les témoignages de ses proches disciples mettent en lumière ces aspects somatiques de l'expérience mystique. L'isolement au sein de la nature, couplé à d'autres exercices spirituels préparatoires, dont, entre autres, la méditation sur les lettres de la Torah, la prière extatique, sont quelques-unes des étapes dans le processus de transformation individuelle. La concentration (hitbodedut) se prolonge par l'union avec les mondes supérieurs, principalement dans la pratique de l'unification (yihud), de l'intention dans la prière (kavvanot) et de l'association avec le divin (devekut) ${ }^{49}$. Une fois les liens avec la matérialité rompus, tout du moins fortement atténués par l'annihilation du moi (bitul ha-yesh), l'humilité, l'élimination des contraintes de la matérialité (katnut) et la projection dans l'immensité du

\footnotetext{
${ }^{47}$ Michel de Certeau, La Fable mystique, XVI - -XVII siècle, Paris, Gallimard, 1982, p. 48-71.

${ }^{48}$ Se prosterner devant des éléments naturels, dont les arbres, les montagnes ou les sources, pour solliciter un bienfait ou provoquer le mal, tendait à diviniser la nature. Cette attitude est dénoncée par Maïmonide dans : «De l'idolâtrie et des coutumes des idolâtres ", Le Livre de la connaissance, Paris, PUF, 1961, p. 219-348. Certains adversaires du hassidisme décelaient, entre autres, dans la sacralisation de la nature, des relents de sabbatianisme. On pense, notamment, à l'idée de devoir descendre dans les tréfonds de la matière, de la réalité physique, voire du mal, pour les sublimer. Ce que les hassidim nomment yerida le-tsorekh aliya (descente pour le besoin de la montée). Voir Rachel Elior, The Mystical Origin of Hasidim, Oxford, Littman Library, 2006, p. 173194.

${ }^{49}$ Sur ces concepts, voir Norman Lamm, The Religious Thought of Hasidism, New York, Yeshiva University Press, 1999.
} 
monde et de l'esprit (gadlut), l'orant connaît des moments de joie, d'exaltation, d'euphorie qui peuvent culminer dans des états paroxystiques. Les textes parlent d'enthousiasme ou d'embrasement (hitlahavut). Autant de notions qui rendent compte d'expériences de type chamanique $^{50}$. L'âme, libérée des chaînes somatiques, s'élance vers les sphères supérieures, à la rencontre du divin. Paradoxalement, la pénétration dans les profondeurs de la psyché suppose une attention extrême à la nature, dans le sens, cette fois, d'états limites de la conscience.

La sortie de soi suppose que le hassid active des pouvoirs, des énergies, des forces qu'il a en lui pour accéder à ces états-limites. Cela exige un changement, une transmutation (shinoui), un renversement (hafikha) de l'individu qui, dépassant l'activité mentale ordinaire, se projette dans une dimension supranaturelle. La déconstruction du sujet social, suivie d'une régénération, d'une restauration (tikkun) suit différentes étapes : en préliminaire, le mystique se sépare du groupe. Il s'isole dans une complète solitude; cette phase liminale de décorporation, de voyage hors $d u$ corps ou d'excursion psychique, permet la dissociation du corps et de l'âme, l'ascension et l'agrégation au monde supérieur, suivi d'une réincorporation, d'une redescente vers le monde réel. Ce qui suppose une interruption momentanée des relations sociales, une suspension de la ritualisation de l'expérience quotidienne, une mise à distance des marques temporelles, afin de se connecter avec les mondes supérieurs. Autant de conditions qui permettent de faire sauter les délimitations entre l'externe et l'interne, le haut et le bas, le somatique et le spirituel. Le mystique peut ainsi se dégager des données de l'expérience quotidienne pour se projeter dans la pure extériorité transcendantale.

Le Besht connut de telles expériences extrêmes lorsque son corps, libéré des pesanteurs terrestres, s'élevait au-delà de la réalité, vers les mondes supérieurs. L'énergie dégagée lors de cette plongée dans les espaces intérieurs, en dehors des habitudes sociales quotidiennes, produit une perturbation du comportement. Cette revitalisation suppose des moments de passivité, de retrait du monde, suivi de phases actives, durant lesquelles les forces accumulées aident à s'associer au divin. La transmutation individuelle peut être soudaine, lente, ou progressive, introduire une altération des états de conscience, dans une forme de

\footnotetext{
${ }^{50}$ Jonathan Garb, Shamanic Trance in Modern Kabbalah, Chicago, Chicago University Press, 2011.
} 
dislocation de la perception, de séparation du monde extérieur et de plongée à l'intérieur de soi, dans l'espace $d u$ dedans. La faculté imaginative prend le dessus et provoque des images, des illuminations qui projettent dans une dimension supranaturelle ou méta-physique. Cette métamorphose mentale implique un déliement, un détachement, une suspension, suivis d'une unification et d'un retour progressif à la normalité. Les hassidim conjuguent dans une multitude de textes un lexique spécifique pour décrire ces états de sortie de la matérialité, d'altération de la conscience et de projection dans une dimension transcendantale. L'idée essentielle est celle de mouvement (hazazah), de renversement (hipukh) ${ }^{51}$, de déplacement (ha'ataqah). Ils parlent aussi du birur, soit une phase de purification du matériel par la séparation et l'élévation des étincelles divines qui sont restées prisonnières de la matérialité et de mesirut nefesh, soit l'abandon des prétentions de l'âme pour devenir une personne autre.

Certes, cette idée de transformation du mystique, de sa nature profonde, existe déjà dans les textes ésotériques. Les hassidim ne font que réactiver des conceptions anciennes qu'on trouve, entre autres, dans la tradition de la littérature des palais, les heykhalot, et dans les écrits kabbalistiques. Elles existent, de plus, dans de nombreuses autres traditions mystiques, notamment asiatiques et islamiques ${ }^{52}$. La grande différence, c'est que, dans le Zohar par exemple, le mystique passe d'un degré à un autre degré, traversant les sept chambres des mondes supérieurs. Il s'agit d'une ascension palier après palier, comparée à une échelle spirituelle ${ }^{53}$. Le Besht tend à démythologiser ce type de description des mondes supérieurs qu'on trouve dans la littérature zoharique. Il ne se focalise pas sur les attributs de Dieu (sefirot) et ne propose pas de description de la topographie sacrée, formée de différentes sphères célestes qui s'emboîtent les unes dans les autres. La cosmogonie kabbalistique cherche avant tout à comprendre le processus de création de l'univers au sein du plérôme. Chez le Besht, on observe, par contre, un recentrement sur l'expérience intérieure. Tout se passe au niveau du sujet qui doit opérer une transmutation intérieure. L'altération de la conscience entraîne, de la sorte, une perception directe, intime et

\footnotetext{
${ }^{51}$ Notons cet usage de la même racine : «Creuse et recreuse la Torah car elle renferme tout. Tourne-la et retourne-la (hafukh ba va-hafukh ba d-kula ba) tout est en elle » (Avot, 8, 21).

${ }_{52}^{52}$ Paul Fenton (éd.), Deux traités de mystique juive, Lagrasse, Verdier, 1987.

${ }^{53}$ Se référer, dans le Zohar, à la description des sept chambres ou palais dans la Parasha Bereshit et dans la Parasha Pekudei.
} 
une association avec le divin. Il s'agit, par des techniques et des exercices spirituels, d'arriver à se projeter au-delà des limites sensorielles ordinaires, de s'introduire dans les territoires les plus profonds de la conscience, tout comme on peut le faire dans le rêve et la prophétie. Le Besht décrit une expérience directe du divin qui, parallèlement à l'étude, la piété, est centrée sur la recherche de l'unification avec les mondes supérieurs. D'où l'insistance sur l'immanence divine, au sein de tous les éléments de la création dont, au premier chef, de la nature. Même si l'essence de la divinité demeure cachée, occultée, voilée, son émanation imprègne toute la création. Rien n'existe qui ne soit animé par l'énergie, le souffle du divin qui s'épanche. Dieu diffuse son énergie, ses influx, ses émanations dans tout ce qui constitue le monde. Il existe une parabole qui rend bien compte de l'acosmisme propre à la pensée du Besht :

Il était une fois un roi sage et grand qui, par un tour de magie, fit apparaître des murs, des tours et des porches. Il ordonna aux hommes de venir chez lui en empruntant ces porches et en passant par ces tours et il exigea que ses trésors soient disposés au coin de ceux-ci. Quelqu'un parvint jusqu'à la première porte, il prit l'argent puis rentra chez lui et d'autres l'imitèrent. Mais son fils bien-aimé fit de grands efforts pour arriver au roi lui-même, jusqu'à son père. Alors il réalisa qu'aucune clôture en vérité ne le séparait de lui, que ces murs et ces porches n'étaient que des illusions. ${ }^{54}$

Contrairement aux textes kabbalistiques, dont le Zohar, qui parlent de degrés, de paliers, d'échelons et de gradations (madregot) entre le monde des humains et le monde céleste ${ }^{55}$, il est ici question d'une élévation de l'âme qui, par l'unio mystica, traverse des cloisons imaginaires pour atteindre les sphères célestes. Il peut, certes, être fait mention d'un rideau (pargod) qui séparerait le monde terrestre et céleste, mais il s'agit d'une séparation symbolique, produit de l'imagination humaine.

Les traditions textuelles se rapportant au Besht décrivent nombre de ces expériences limites. Il est question d'une extraction de l'âme de la routine quotidienne, d'un voyage dans les espaces intérieurs jusqu'à Dieu, suivi d'une redescente, certes transformé, vers la réalité terrestre ${ }^{56}$. L'isolement au sein de la nature est couplé à d'autres exercices spirituels

\footnotetext{
${ }^{54}$ Keter Shem Tov, New York, Kehot, 2004, p. 31, n 51.

${ }^{55}$ Voir dans le Zohar la Parasha Pekudei qui décrit les sept palais du monde divin.

${ }^{56}$ Voir l'Iggeret ha-Kodesh, récit d'un voyage du Besht vers les mondes supérieurs et sa rencontre avec le Messie. Jean Baumgarten, Naissance du hassidisme, Paris, Albin Michel, 2006, p. 249-258.
} 
préparatoires. La marche, par exemple, peut être une première étape dans un processus de transformation individuelle, sorte d'épreuve de repentir (teshuva) reliée à l'expiation des fautes et à la pensée de l'exil. Les textes décrivent, de même, le fait de tomber à terre, prostré, la tête entre les genoux, en position quasi fotale (nefilat appayim). Il s'agit de quitter la verticalité, de se baisser, de s'humilier devant Dieu, dans une posture d'auto-annihilation qui précède la transe proprement dite, signe de l'illumination et de la montée de l'âme (aliyat ha-neshamah). On atteint ainsi le rétrécissement, le désinvestissement de la corporéité (hitpashtut ha-gashmiyut). Le mystique ôte de lui toutes les pensées parasites et, par la faculté imaginative, se transporte et se meut dans l'immensité cosmique. Une fois les liens avec la matérialité rompus, l'orant ressent une grande euphorie qui peut culminer dans des états paroxystiques.

Paradoxalement, la pénétration dans les profondeurs de la psyché suppose une attention extrême à la nature, aux états altérés de la conscience. Le terme le plus usité est l'embrasement mystique (hitlahavut). Le ravissement (hitpa'alut) désigne plus particulièrement l'effet qu'un tel enflammement produit sur l'orant qui, perdant toute retenue somatique, est envahi par des mouvements désordonnés, signe de l'intensité dans sa recherche de Dieu, fait d'un mélange de crainte et de joie intenses.

En yiddish, on dit s'brent, allusion au feu qui enflamme l'âme du mystique, entouré d'une soudaine luminosité. Le processus de transformation intérieure est parfois représenté par la différence entre le bois sec et le bois brûlé qui a subi une consumation. L'annihilation de la réalité extérieure, tel un feu qui embrase et transforme, permet de transpercer l'enveloppe de la matérialité pour se projeter dans une dimension extra-sensorielle ${ }^{57}$. Elle s'accompagne d'une forme d'ubiquité, de capacité de se mouvoir dans les espaces du dedans. Les textes du Besht parlent d'évanouissement, d'endormissement durant une première phase de déprise de la matérialité, assimilée à un évanouissement (en yiddish, khalushes) ou à une mort mystique. Il existe des formes passives, sorte de catalepsie, d'immobilité de pierre, de statisme, d'anesthésie, dans lesquels le corps est en veilleuse. D'autres fois, le mystique tombe à terre les membres tendus, accompagnés de mouvements désordonnés, de pleurs, voire de borborygmes incompréhensibles. La glossolalie, la parole automatique, témoigne que

\footnotetext{
${ }^{57}$ Sur l'expérience mystique comparée à un feu qui consume, voir Jonathan Garb, Shamanic Trance, op. cit., 2011, p. 36-45.
} 
la shekhinah parle par la bouche du mystique. La perte de conscience se traduit, dans les cas les plus extrêmes, par des signes corporels proches de la transe chamanique ${ }^{58}$.

Un des traits distinctifs du hassidisme, dans cette perspective d'exploration des états limites de la nature humaine, reste la prière extatique. Elle tranche radicalement avec les modes de louange divine propres à la tradition rabbinique dans laquelle l'orant adresse avant tout une supplique à Dieu dont il sollicite l'intercession et la protection. La prière hassidique comprend, certes, ces dimensions, mais elle vise avant tout à l'union avec la présence divine à travers la méditation, la concentration et la suspension des liens avec le monde matériel. De plus, l'orant n'est pas censé prier à des moments fixes; il prie quand il sent que son âme est prête, mue par une intense ferveur et une intention pure, selon le principe kabbalistique des kavvanot. Il se concentre sur les mots de la prière comme stimulant de l'expérience extatique. Ce qui n'implique aucune limitation, ni empêchement, mais un relâchement des obstacles matériels qui peuvent entraver l'ascension de l'âme. Cet état d'exaltation intense ${ }^{59}$, conséquence de la séparation de la matérialité, est comparé soit à une petite mort, à une ivresse incontrôlable, soit à une forme d'union érotique avec le divin :

Quand l'orant s'est dépouillé de toute corporéité, de toute forme de volonté, qu'il est lié uniquement au Créateur, qu'il ne sait plus comment réciter ses prières en raison de l'éblouissement dans lequel il est plongé et de l'association avec Dieu, il récite tout de même ses prières dans le bon ordre. Les cieux ont pitié de lui, lui donnent le pouvoir de parler et de prier, comme il est dit (Ps. 51, 17) : Seigneur, puisses-tu m'ouvrir les lèvres, pour que ma bouche proclame tes louanges $!^{60}$

De multiples récits décrivent ces états de transe durant la prière. Abraham de Kalisk fut, par exemple, accusé par les autorités de Vilna de sauter devant l'arche et d'être agité de mouvements désordonnés ${ }^{61}$. Il en

\footnotetext{
${ }^{58}$ Sur la transe et les comportements religieux extrêmes (ekstasis, kinesis, furor, stupor, raptus, mania), fondés sur l'augmentation des stimulations sensorielles, l'altération de la conscience, la perte de contrôle, suite à l'expérimentation de pratiques, rituels et techniques mystiques, voir Wouter Hanegraff, «trance », Vocabulary for the Study of Religion, Robert A. Segal and Kocku von Stuckrad (ed.), Leiden, Brill, 2015, p. 511513.

${ }^{59}$ Rivka Schatz Uffenheimer, « Anarchic Manifestations in Hasidic Life », Hasidism as Mysticism, Jérusalem, Magnes ; Princeton, Princeton University Press, 1993, p. 242254.

${ }^{60}$ Israël Shabbetai Koznitz, Avodat Israel, Munkacz, 1929, p. 51 b.

${ }^{61}$ Elijah Judah Schochet, The Hasidic Movement and the Gaon of Vilna, Northvale, Aronson, p. 52.
} 
va de même avec Levi Yitshak de Berditchev qui était connu pour son intense mobilité fébrile durant la prière. On raconte que lorsqu'il récitait le début de la prière Barukh Atah Adonaï (Bénis sois-tu Adonaï), il prononçait à haute voix les mots atah, atah, atah dans un état d'exaltation extrême. La concentration sur certains mots de la prière le transportait, souvent pendant de longs moments, dans un état de grande ferveur, entrecoupé de convulsions, signe que son âme était propulsée dans les sphères supérieures.

On raconte encore qu'il perdait à ce point le sens du réel qu'il pouvait passer sa main à travers du verre sans rien sentir, tenir sa paume longtemps au-dessus d'une flamme, danser, renverser des tables, les plats, sans avoir l'impression de commettre des actes déplacés. Hayyim de Sancz raconte que Levi Yitshak de Berditchev «tremblait d'effroi devant Dieu même dans son sommeil. Endormi, la crainte de Dieu le faisait frissonner comme n'importe quelle personne qui serait saisie de peur entourée de toutes parts par des voleurs ${ }^{62}$. On le décrit les cheveux hérissés, se tenant tantôt prostré, tantôt traversé de mouvements saccadés incontrôlables, passant d'un coin d'une pièce à une autre, au point de faire peur aux juifs en train de prier à côté de lui et d'ameuter ses adversaires pour constater qu'il était saisi par l'esprit de folie. Un opposant vint un jour l'observer; Levi Yitshak de Berditchev le saisit et le trimballa d'un bout à l'autre de la synagogue. Si bien que celui-là finit par croire ce qu'on lui avait raconté : le rebbe était dans un état de transe extatique qui lui faisait perdre tout contrôle, preuve de l'intensité de sa ferveur. Une fois, en puisant de l'eau pour cuire des matsot à Pessah, il fut saisi de tremblements et faillit tomber dans le puits. Il lui arrivait, en lisant à Purim la Megilat Esther de danser devant l'Arche sainte ${ }^{63}$. On pourrait également citer Shne'ur Zalman de Lyadi qui utilise la métaphore du guerrier qui attaque le mal d'où son ardeur dans la prière, comparable à celle d'un combattant sur un champ de bataille ${ }^{64}$. On pense surtout à Nahman de Bratslav qui décrit de telles plongées dans les espaces du dedans et les manifestations convulsives, les mouvements exubérants et l'allégresse physique qui en découlent. Un extrait explique que, vers la fin de sa vie, Nahman aimait s'entourer de gens avec qui il

\footnotetext{
${ }^{62}$ Toldot Kedushat Levi, éd. par Tsevi Elimelekh Kalish, Munkacz, 1938, fol. 153, $\mathrm{n}^{\circ} 50$.

${ }^{63}$ Sur ces états de transe extatique durant la prière chez Levi Yitshak de Berditchev, voir Israel Berger (ed.), Eser Orot, Pietrikov, Kleiman, 1907, III, 3-9.

${ }^{64}$ Cité par Louis Jacobs, Hasidic Prayer, London, Littman Library, 1993, p. 99.
} 
entamait des discussions sur des sujets spirituels et à qui il racontait des contes :

Une des caractéristiques notables reste que, même quand le Rebbe parlait aux gens de choses profanes (sihot hulin), ses jambes et presque tout son corps tremblaient. Ses jambes étaient littéralement agitées par des secousses, quasi en état de commotion. S'il était assis à une table avec des gens, la table et tous les gens se mettaient à trembler. Ses jambes et son corps tremblaient et ils étaient constamment pris de convulsions. Quiconque n'a pas contemplé la crainte de Dieu sur son visage n'a jamais vu la crainte de Dieu de toute sa vie. Il était rempli. ${ }^{65}$

Cette force de combustion, d'inflammation, de ferveur intense, témoigne de l'ascension de l'âme du mystique vers les mondes supérieurs et de la circulation des influx divins entre le monde d'en haut et le monde d'en bas; les énergies drainées vers la terre sont ensuite diffusées horizontalement par le tsaddik, messager et ambassadeur des mondes supérieurs, qui, après son retour sur terre venant de l'au-delà, propage les émanations vers les disciples. À l'aspect individuel, notamment dans la prière extatique, s'ajoute une dimension collective, autour du rebbe qui, durant le repas pris en commun (tish), le troisième repas du Shabbat (se'udah ha-shlishit) et lors des bénédictions collectives, fait circuler les énergies au sein de sa communauté. Il crée ainsi une sorte de confraternité mystique qui préfigure la société d'après la venue du Messie. Le pouvoir télépathique du rebbe lui permet de communiquer avec ses adeptes au-delà du langage, soit lors d'un contact personnel (yihud), soit par une forme de télépathie collective au sein de la cour hassidique. Nahman de Bratslav compare le tsaddik à un père attentionné ou à une mère qui allaite ses enfants ${ }^{66}$.

Un autre aspect original de la pensée hassidique, fondée sur une dialectique vivante entre l'être (gadlut) et l'étant (katnut), le vide infini (ayn) et la matière, l'ipséité (yesh), les sphères supérieures et le monde terrestre, concerne le service divin par la corporéité (avoda begashmiyut). Elle suppose une complémentarité constante entre la carnalité, la physicalité, les données somatiques et les réalités spirituelles. Dès l'instant où le divin imprègne toutes les dimensions de la vie, de la nature, du monde, la dichotomie entre le corps et l'esprit est remise en

\footnotetext{
${ }^{65}$ Nahman de Bratslav, Hayyei Moharan, Jérusalem, Torat ha-netsah, n ${ }^{\circ 42}$. Voir aussi à ce sujet Zvi Mark, op. cit., 2009, p. 135-137.

${ }^{66}$ La conception mystique du tsaddik, entre autres, père et mère de ses disciples, est exposée dans Nahman de Bratslav, Likkutei Moharan, op. cit., I, 4.
} 
cause. Toute activité matérielle comporte une dimension divine et doit, de ce fait, non pas être refoulée mais, au contraire être exaltée, sanctifiée. Chaque parcelle de la réalité comprend des graines ou des étincelles (nitsotsot) de sainteté qui doivent être libérées, comme on le trouve explicité dans le Testament du Besht :

C'est un principe important que lorsque l'être humain se vêtit, mange ou fait usage d'une chose, il tire bénéfice de la force vitale qui se trouve dans cette chose. L'objet ne peut pas exister sans cette composante spirituelle. Il contient des étincelles saintes (nitsotsot) qui sont liées à la racine de son âme. J'ai entendu dire que c'est la raison pour laquelle une chose est prisée par certains et détestée par d'autres. Quand on fait usage d'une chose, qu'on mange, même si c'est pour un besoin physique, tu rectifies ces étincelles. Elles sont rectifiées du fait que tu utilises la force ajoutée à ton corps par le vêtement, la nourriture ou par toute autre chose, dans le but de servir Dieu... Israel Baal Shem Tov, que la paix soit sur lui, avait ainsi l'habitude de dire: Quand une personne mange, s'assied avec d'autres personnes et utilise les autres, cela signifie qu'il s'occupe des étincelles dans les choses. Toute personne doit, de ce fait, être concernée par ses objets et par tout ce qu'il possède du fait des étincelles qu'ils contiennent; il doit être préoccupé par les étincelles saintes (qui imprègnent les choses). ${ }^{67}$

D'où le paradoxe de la doctrine hassidique axée sur l'annihilation du monde matériel et, en même temps, l'exaltation du corps, lieu de rencontre du divin, tremplin vers les sphères célestes et plaque sensible qui réverbère les volontés supérieures. Tout le monde peut accomplir une unification (yihud) avec la divinité lors d'une banale action, ou lors de toute activité, comme faire des affaires, converser de choses profanes (sihot hulin), manger, boire, avoir une relation sexuelle. Dans la tradition éthique, notamment dans les Devoirs du cour d'Ibn Pakuda ${ }^{68}$, manger, boire et dormir sont considérés comme un moyen de renforcer son corps pour le service du Créateur. Pour les hassidim, cela permet, de même, en référence à la kabbale lurianique, d'extraire et d'élever les étincelles saintes qui sont contenues dans la nourriture. Le Besht sacralise les activités matérielles, physiques et somatiques, de même qu'il humanise le divin.

Ces quelques brefs fragments nous rappellent que la doctrine hassidique se définit, entre autres, par l'attention portée à la nature et à l'exploration des potentialités psychiques et psychologiques de la nature humaine, en vue de la louange divine et de l'union au Très Haut. Ces

\footnotetext{
${ }^{67}$ Tsava'at ha-ribash, op. cit, 1998, 109.

${ }^{68}$ Chapitre sur le service divin.
} 
aspects ont été longtemps négligés par les chercheurs, qui ont centré leurs études sur la détermination des catégories anthropologiques et des principes de la pensée religieuse des maîtres, en oubliant l'importance de la magie, de la nature, du corps et des exercices mentaux destinés à accroître les potentialités spirituelles des individus aux fins de convoquer les anges, les prophètes, et de se rapprocher de Dieu. 